\title{
A Study of MODS in Dengue Fever with Emphasis on Comparison between Survivors \& Non-Survivors
}

\author{
Kiran H.S', Ravi Kumar Y.S', Subhaschandra B.J ${ }^{3}$ \\ ${ }^{1}$ Associate Professor, Department of Internal Medicine, JSS Medical College and Hospital, JSSAHER, Mysore, Karnataka, India. ${ }^{2}$ Professor, Department of \\ Internal Medicine, JSS Medical College and Hospital, JSSAHER, Mysore, Karnataka, India, ${ }^{3}$ Professor \& HOD, Department of Internal Medicine, JSS \\ Medical College and Hospital, JSSAHER, Mysore, Karnataka, India.
}

\section{Abstract}

Background: Dengue is the most common mosquito-borne viral disease throughout the world. Dengue could be asymptomatic or it may manifest as non-severe, severe and fatal types. Severe dengue manifests itself in myriad ways. Excessive hospitalization of dengue patients occurs due to the absence of definite early prognostic markers or early predictors of severity/mortality. There are few Indian studies on mortality in severe dengue. The objective of this study was to explore Multi-organ dysfunction syndrome (MODS) in Dengue fever with an emphasis on comparison between survivors \& non-survivors. Subjects and Methods: This study was done at JSS Hospital, a tertiary referral teaching hospital of JSSMC, JSSAHER with 80 adult ICU beds. It was a retrospective observational study. Patients with laboratory-confirmed severe dengue infections satisfying inclusion and exclusion criteria and admitted to the ICU between September 2016 and September 2017, including the large outbreak period were considered. The data were collected from inpatient case-records and the analysis was done, retrospectively. Results: A total of 29 patients met all inclusion and exclusion criteria. In our study, out of 29 cases, total number of males were $17(58.6 \%)$, whereas females were $12(41.4 \%)$. Mean age of patients was 43.37 years (SD:14.36). The mortality of severe dengue with MODS admitted to the ICU remains high $(55.2 \%)$ in this study. In this study, mean age was more in survivors when compared to non survivors; At admission, Creatinine, Lactate \& PCV were higher among non survivors; All patients with Hypotension, Both the patients with ARDS, myocarditis \& All the 6 patients who had bleeding manifestations expired \& prolonged a PTT and PT-INR were found among many non survivors; Other parameters like LFT, Se.Albumin and Comorbidities did not have any impact on mortality. In this study, though both the groups had hypoalbuminemia, Serum Albumin was not statistically significant between survivors \& non-survivors while arterial Lactate, creatinine and PCV were significant. Conclusion: The mortality of severe dengue with MODS admitted to the ICU remains high. In this study, surprisingly, mean age was more in survivors when compared to non-survivors; Creatinine, Lactate \& PCV were higher among non-survivors. Other parameters like LFT, Se.Albumin and Comorbidities did not have any impact on mortality. Definite early prognostic markers or early predictors of severity/mortality is the need of the day.

Keywords: Dengue fever, Multi-organ failure (MOF), Multi-organ dysfunction syndrome(MODS).

Corresponding Author: Dr. Kiran H.S, Associate Professor, Department of Internal Medicine, JSS Medical College and Hospital, JSS AHER, Mysore, Karnataka, India.

Received: May 2019

Accepted: May 2019

\section{Introduction}

Dengue is the most common mosquito-borne viral disease throughout the world. ${ }^{[1]}$ Dengue has affected nearly 390 million people annually leaving nearly 20,000 dead every year. ${ }^{[2]}$ The incidence of dengue has risen exponentially in the last 50 years affecting patients in more than 100 endemic countries (risking nearly $50 \%$ of the global population). ${ }^{[3]}$ The South-East Asia is hyperendemic with rise in dengue cases since 2000. With the onset of rainy season (monsoon) and thereafter, various areas of India go through epidemics of dengue every year. ${ }^{[4]}$ Areas in \& around Mysore went through a very severe epidemic in 2017. Dengue is a dynamic, systemic, infectious disease. ${ }^{[5]}$ Dengue could be asymptomatic or it may manifest as non-severe, severe and fatal types. ${ }^{[6-8]}$ Severe dengue manifests itself in myriad ways. Mortality in severe dengue is related to multiple organ dysfunctions. Excessive hospitalization of dengue patients occurs due to the absence of definite early prognostic markers or early predictors of severity/mortality. ${ }^{[9]}$ There are few Indian studies on mortality in severe dengue. The objective of this study was to explore Multi-organ dysfunction syndrome (MODS) in Dengue fever with an emphasis on comparison between survivors \& non-survivors.

\section{Subjects and Methods}

This study was done at JSS Hospital, a tertiary referral teaching hospital of JSSMC, JSSAHER with 80 adult ICU beds. It was a retrospective observational study. Patients with laboratory-confirmed severe dengue infections and admitted to the ICU between September 2016 and September 2017, including the large outbreak period were considered. The 
data were collected from inpatient case-records and the analysis was done, retrospectively. Inclusion criteria were: Adults > 18 years of age, confirmed diagnosis of Dengue Infection by IgM (ELISA) or NS-1 antigen (ELISA) and final diagnosis of Dengue associated MODS/MOF. Exclusion Criteria were Confounding diagnosis (concurrent Malaria /Leptospirosis /Rickettsial/Bacterial infections).

The medical records of all the enrolled patients were studied for the following details: age, gender, clinical manifestations, underlying conditions, laboratory investigations at admission and outcomes; Data was entered in Microsoft EXCEL software \& analyzed using the software Epi Info $^{\text {TM }}$ for Windows version 7.2 (https://www.cdc.gov/epiinfo/pc.html); Student t test was used for comparing two groups; $\mathrm{P}$ value < 0.05 was considered as significant.

\section{Results}

A total of 29 patients met all inclusion and exclusion criteria. In our study, out of 29 cases, total number of males were 17 $(58.6 \%)$, whereas females were $12(41.4 \%)$. Mean age of patients was 43.37 years (SD:14.36). Out of 29 patients, 18 (62\%) were positive for NS1 Antigen, 8 (27.5\%) were positive for IgM ELISA, and $3(10.5 \%)$ were positive for both.

Fever was the main presenting symptom $(100 \%)$ in all our patients followed by vomiting $(41.4 \%)$, headache/retroorbital pain $(34.5 \%)$, myalgia $(6 \%)$, breathlessness $(10 \%)$, altered sensorium (17.2\%), bleeding manifestations $(20.6 \%)$, arthralgia (6.8\%), and diarrhea (10.3\%). Clinical examination revealed Icterus in 4 patients (13.8\%), Hypotension in 15 patients $(51.7 \%)$.

Bleeding manifestations were seen in 6 patients (gum bleeding-4 patients, Hemetemesis in 2 patients \& Hematuria in 2 patients). Ultrasonographic evaluation revealed $b / 1$ pleural effusion in 9 patients $(31 \%)$, Ascites+pleural effusion in 5 patients $(17.2 \%)$ and polyserositis in 4 patients $(13.8 \%)$. A few had hepato-splenomegaly, in combination or alone. CXR was consistent with ARDS in 2 patients \& ECHO was suggestive of myocarditis in 2 patients. A few patients had co-morbidities (underlying conditions like both DM \& HTN in 2 patients, both HTN \& IHD in 1 patient, DM, HTN \& Rheumatoid arthritis in 1 patient, only DM in 2 patients, 1 patient who had CKD was also HCV positive, 1 patient had TOF \& 1 patient was an alcoholic). All patients except one had thrombocytopenia of varying degrees (ranging from 1000 to $1.24 \mathrm{~L}$ ) and no significant difference was noted between survivors and non-survivors.

Out of the 29 patients, 27(93.1\%) had altered LFT - Mean Total Bilirubin:4.04(SD:3.37)mg/dl, Mean AST:648.18 U/L, Mean ALT:414.11U/L,Mean ALP:408.71U/L. Prothrombin time (PT) was elevated in 7 patients $(24.1 \%$ ) and aPTT in 6 patients(20.7\%). 10 patients(34.5\%) had AKI-Mean Blood Urea:72.04(SD:57.97)mg/dl \& Mean Se.Creatinine:2.02(SD:1.59)mg/dl. ABG showed Metabolic Acidosis in 14 patients (48.3\%). 19 patients (65.5\%) had elevated Lactate - Mean Lactate: 5.88(SD: 4.06). Mean Albumin was 3.04(SD: 1.03) g/dl. Out of 29 patients, $22(75.9 \%)$ had hypoalbuminemia. Mean $\mathrm{Hb} \%$ was $13.2 ; 1$ patient had $\mathrm{Hb} \%$ of $18.4 \& 3$ patients had $\mathrm{Hb} \%$ between 17
\& 17.5. Mean PCV\% was 41.9; 1 patient had PCV >60, 1 patient had PCV between $55-60 \& 5$ patients had PCV between 50-55.

Out of 29 patients, 16 patients died (55.2\%), 2 patients $(6.8 \%)$ got discharged against medical advice and 11 patients $(38 \%)$ improved and were discharged. 18 patients $(62 \%)$ needed intubation. Mean ICU stay was 3 days.

\section{Outcome of MODS in Dengue}
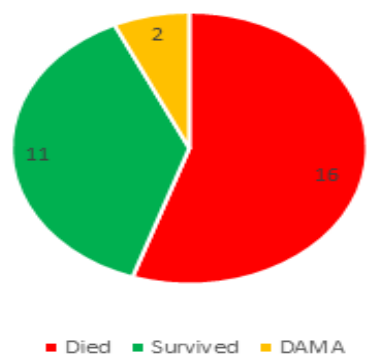

TOTAL no.of cases- 29

SURVIVORS- 11

NON SURVIVORS- 16

DAMA- 2 (Discharged against medical advice)

\begin{tabular}{|c|c|c|c|}
\hline \multicolumn{4}{|c|}{ Comparison of Survivors \& Non Survivors: Mean (S.D) } \\
\hline & $\begin{array}{l}\text { Survivors } \\
(n=11)\end{array}$ & $\begin{array}{l}\text { Non survivors } \\
(n=16)\end{array}$ & P value \\
\hline Age in yrs & $50.91(11.80)$ & $37.38(14.33)$ & 0.01 \\
\hline Blood Urea (mg/dl) & $48.27(35.55)$ & $77.87(62.44)$ & 0.14 \\
\hline Se.Creatinine $(\mathrm{mg} / \mathrm{dl})$ & $1.2(0.63)$ & $2.42(1.9)$ & 0.03 \\
\hline Total Bilirubin $(\mathrm{mg} / \mathrm{dl})$ & $3.31(3.15)$ & $4.66(3.75)$ & 0.33 \\
\hline AST (U/L) & 424.36 & 893.07 & 0.32 \\
\hline ALT (U/L) & 305.64 & 543.13 & 0.39 \\
\hline ALP (U/L) & 449.45 & 399.2 & 0.69 \\
\hline $\mathrm{Hb} \%(\mathrm{gm} / \mathrm{dl})$ & $12.35(2.08)$ & $13.73(2.29)$ & 0.12 \\
\hline PCV $(\%)$ & $36.6(7.96)$ & $45.63(7.97)$ & 0.009 \\
\hline Se.Albumin (gm/dl) & $3.04(0.49)$ & $2.9(1.3)$ & 0.84 \\
\hline Lactate & $3.67(2.21)$ & $7.22(3.8)$ & 0.02 \\
\hline
\end{tabular}

\section{Age of Survivors \& Non survivors}
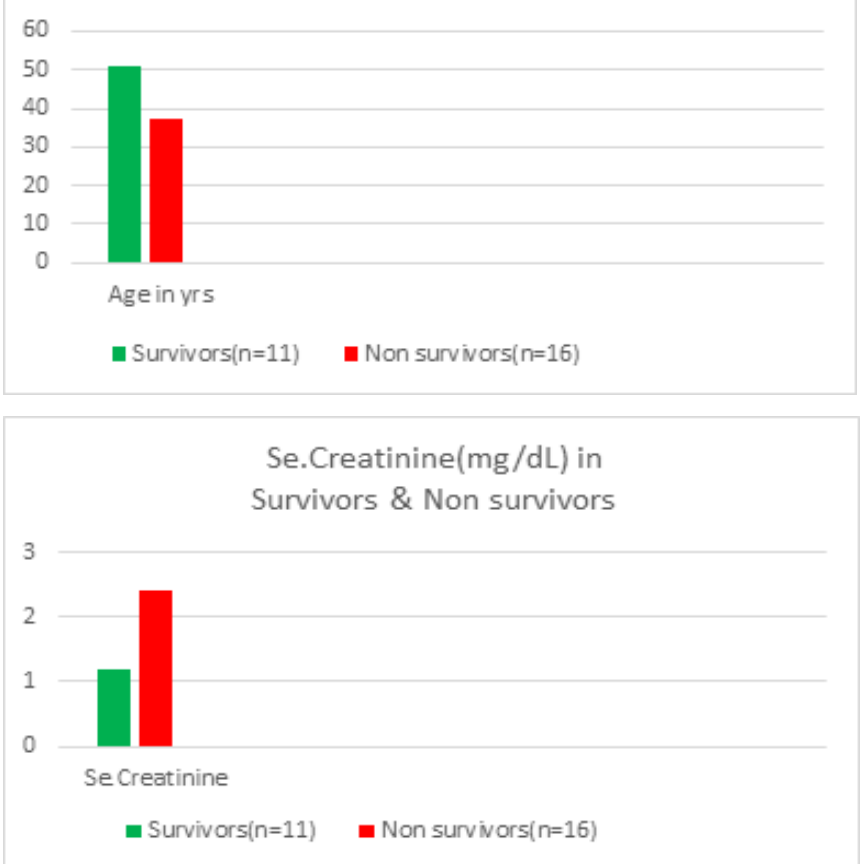


\section{PCV in Survivors \& Non survivors}

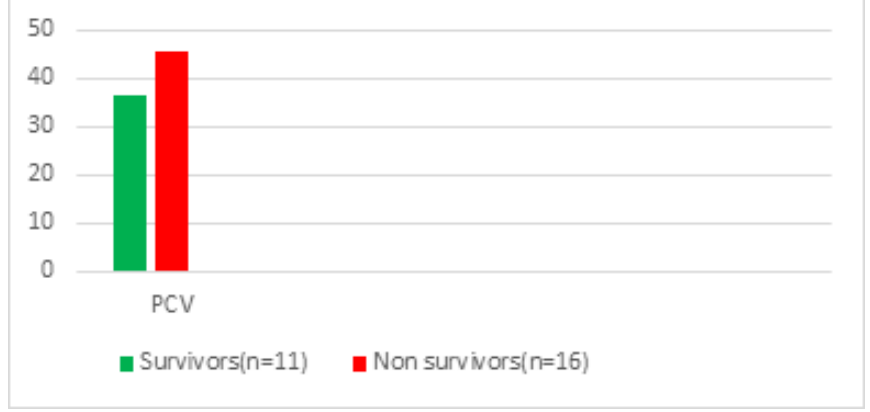

Lactate in Survivors \& Non survivors

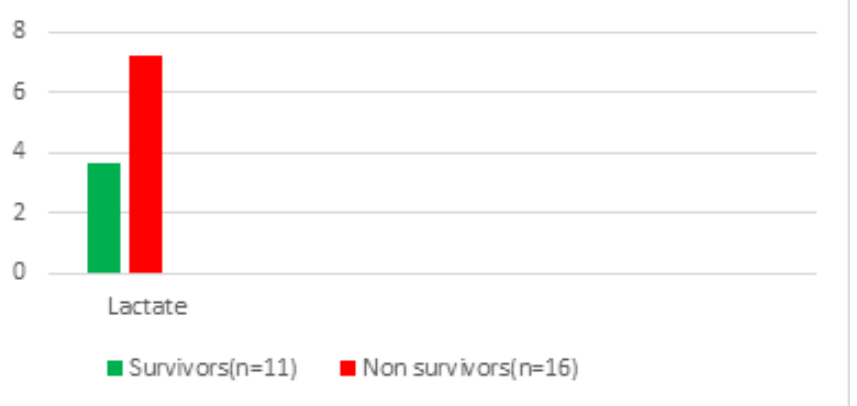

\section{Discussion}

The mortality of severe dengue with MODS admitted to the ICU remains high $(55.2 \%)$ in this study. In this study, mean age was more in survivors when compared to non survivors; At admission, Creatinine, Lactate \& PCV were higher among non survivors; All patients with Hypotension, Both the patients with ARDS, myocarditis \& All the 6 patients who had bleeding manifestations expired \& prolonged aPTT and PT-INR were found among many non survivors; Other parameters like LFT, Se.Albumin and Comorbidities did not have any impact on mortality. In this study, though both the groups had hypoalbuminemia, Se.Albumin was not statistically significant between survivors \& non-survivors while arterial Lactate was significant whereas both were significant in the study by Jog et al. ${ }^{[10]}$ These parameters could be taken as prognostic markers \& predictors of mortality. In this study, surprisingly, mean age was more in survivors when compared to non-survivors which is diametrically opposite to the study by Hsieh et al. ${ }^{[11]}$ A better immunity due to previous exposures could be a likely explanation for this phenomenon of age being a favorable factor for surviving severe dengue seen in our study. In this study, abnormal LFT results were seen in non-survivors compared with survivors but the difference was not statistically significant which concurs with similar findings by Karunakaran et al. ${ }^{[12]}$ This study shows that Lactate levels were higher among non-survivors which is consistent with other studies. ${ }^{[13]}$ There was no significant difference in platelet counts between survivors and non-survivors which concurs with the study by Siddharth Jain et al. ${ }^{[14]}$ This study shows that Creatinine levels were higher among nonsurvivors which is consistent with other studies. ${ }^{[11]}$ Hemoconcentration is a well-known finding of Dengue. ${ }^{[15,16]}$ This can be explained by the concentration of plasma due to fluid leakage, which causes a rise of haemoglobin weight in a unit volume of blood. When plasma leakage through the blood vessels occurs, PCV/ hematocrit value is also expected to rise. ${ }^{[17,18]}$ This study shows that PCV was higher among non-survivors.

Limitations of this study: This is a single centre retrospective study. Hence, a large multicenter prospective study is desirable to evolve better predictors of mortality.

\section{Conclusion}

The mortality of severe dengue with MODS admitted to the ICU remains high. In this study, surprisingly, mean age was more in survivors when compared to non-survivors; Creatinine, Lactate \& PCV were higher among nonsurvivors. Other parameters like LFT, Se.Albumin and Comorbidities did not have any impact on mortality. Definite early prognostic markers or early predictors of severity/mortality is the need of the day.

\section{Acknowledgment:}

We express our heartfelt gratitude to our Respected Principal and the Medical Records Department

\section{References}

1. Murray NE, Quam MB, Wilder-Smith A. Epidemiology of dengue: past,present and future prospects. ClinEpidemiol 2013;5:299-309.

2. Bhatt S, Gething PW, Brady OJ, Messina JP, Farlow AW, Moyes CL, et al.The global distribution and burden of dengue. Nature. 2013; 496: 504-507.

3. World Health Organization.What is dengue? Available from:https://www.who.int/denguecontrol/disease/en/epidemiology[Las $\mathrm{t}$ accessed on 2019 Jan 19].

4. Kamath S, Jain N, Gupta, Jha AC, Rao BS. Dengue Epidemic in Jamshedpur-Tata Main Hospital (TMH) Experience. J Trop Dis 2015;3:159.

5. Guzman MG, Gubler DJ, Izquierdo A, Martinez E, Halstead S. Dengue infection. Nat Rev Dis Primers. 2016; 16055

6. Pan American Health Organization. Dengue: Guidelines for patient care in the region of the America. 2nd edition. Washington DC: Paho. 2016.

7. Guzman MG, Harris E. Dengue. Lancet 2015;385:453-65.

8. Halstead SB. Dengue. Lancet 2007;370:1644-52.

9. Lee L K, Earnest A, Carrasco LR, Thein TL, Gan VC, Lee VJ,etal.Safety and cost savings of reducing adult dengue hospitalization in a tertiary care hospital in Singapore. Trans R Soc Trop Med Hyg 2013; 107: 37-42.

10. Jog et al. Dengue infection with multiorgan dysfunction:-sofa score, arterial lactate and serum albumin levels are predictors of outcome Intensive Care Medicine Experimental 2015 3(Suppl 1):A830.

11. Hsieh CC, Cia CT, Lee JC, Sung JM,Lee NY, Chen PL, et al. A Cohort Study of Adult Patients with Severe Dengue in Taiwanese Intensive Care Units: The Elderly and APTT Prolongation Matter for Prognosis. PLoSNegl Trop Dis. 2017; 11(1): e0005270.

12. Karunakaran A, Ilyas WM, Sheen SF et al Risk factors of mortality among dengue patients admitted to a tertiary care setting in Kerala, India. J Infect Public Health 2014; 7:114-20.

13. Thanachartwet V, Wattanathum A, Oer-Areemitr N, Jittmittraphap A, Sahassananda D, Monpassorn C, Desakorn V. Diagnostic accuracy of peripheral venous lactate and the 2009 WHO warning signs for identifying severe dengue in Thai adults: a prospective observational study. BMC Infect Dis. 2016;16(1)

14. Jain S, Mittal A, Sharma SK, et al. Predictors of Dengue-Related Mortality and Disease Severity in a Tertiary Care Center in North India. Open Forum Infect Dis. 2017;4(2):ofx056. 
15. WHO . Comprehensive guidelines for prevention and control of dengue and dengue haemorrhagic fever. Geneva: WHO; 2011.

16. Handbook for clinical management of dengue - World Health Organization

2012. http://www.wpro.who.int/mvp/documents/handbook_for_clinical_man agement_of_dengue.pdf.(Accessed 19 Jan 2019)

17. Tee HP, How SH, Jamalludin AR, FarizSafhan MN, MohdSapian M,
Kuan YC, et al. Risk factors associated with development of dengue haemorrhagic fever or dengue shock syndrome in adults in Hospital TengkuAmpuanAfzanKuantan. Med J Malaysia. 2009;64:316-320.

18. Kularatne SAM, Weerakoon KGAD, Munasinghe R, Ralapanawa UK, Pathirage M. Trends of fluid requirement in dengue fever and dengue haemorrhagic fever: a single centre experience in Sri Lanka. BMC Res Notes. 2015;8:130.

Copyright: (C) the author(s), 2019. It is an open-access article distributed under the terms of the Creative Commons Attribution License (CC BY 4.0), which permits authors to retain ownership of the copyright for their content, and allow anyone to download, reuse, reprint, modify, distribute and/or copy the content as long as the original authors and source are cited.

How to cite this article: Kiran HS, Kumar YSR, Subhaschandra BJ. A Study of MODS in Dengue Fever with Emphasis on Comparison between Survivors \& Non-Survivors. Acad. J Med. 2019;2(1):08-11.

DOI: dx.doi.org/10.21276/ajm.2019.2.1.3 